\title{
Additional symmetries of KP, Grassmannian, and the string equation II
}

\author{
L.A.Dickey \\ University of Oklahoma, Norman, OK 73019 \\ e-mail: ldickey@nsfuvax.math.uoknor.edu
}

October, 1992

1. Introduction. As in the first part of this paper (see [1]) we are interested in the connection between additional symmetries and the string equation. Now we extend this theory to the multi-component (i.e. matrix) KP or $\mathrm{KdV}(\mathrm{mcKdV})$ hierarchies, and to modified $\mathrm{KdV}$ $(\mathrm{mKdV})$.

In addition to having pure mathematical interest, this study is also necessary for some physical applications. It is known that some matrix models, such as e.g. unitary matrix models, lead to the string equation for mKdV (see [10] and [2]). An important feature of a string equation is its close relation to some hierarchy of integrable equations. This is wellknown in the case of the string equation of $\mathrm{KdV}$ hierarchies. The relation between them consists in the fact that the string equation is invariant under the flows generated by the hierarchy equations. As the experience with $\mathrm{KdV}$ shows, the origin of this invariance is the following: there are some additional symmetries of the hierarchy which do not belong to the hierarchy although they commute with it. The set of fixed points of an additional symmetry is invariant under the hierarchy, and the string equation is nothing but the condition of this stationarity.

Thus, we would study additional symmetries acting on $\mathrm{mKdV}$ if not for a little obstacle: to all appearances, the additional symmetries do not act on $\mathrm{mKdV}$ at all! How can this happen if it is known that they act on KdV hierarchies, and the latter are connected with $\mathrm{mKdV}$ by the Miura transformation? The problem is that there are, in fact, $n$ different $\mathrm{KdV}$ operators corresponding to the same mKdV operator, and the results of action of the additional symmetry on them are not compatible (this is only our conjecture but a very plausible one).

Fortunately, the mKdV equation can be embedded into the mcKdV hierarchy (we are not sure if this fact has been somewhere mentioned before). In this, wider, phase space the additional symmetries do not act worse than they did for the ordinary, scalar KdV. It is true that the flows of additional symmetries do not respect the submanifold representing mKdV. But if a point on the submanifold is stationary under an additional symmetry, it stays on this submanifold. The mKdV string equation induces the corresponding KdV string equation.

Irrespectively of their application to $\mathrm{mKdV}$, the $\mathrm{mcKdV}$ can play a role in some matrix models. This is why we start from these hierarchies. In contrast to [10] or [2] we consider 
hierarchies of an arbitrary order $n$ (instead of $n=2$ ): consideration of a special case often obscures general facts and formulas.

Here, as well as in the first part, we are prompted by a desire to show that the notion of an additional symmetry which, only a few years ago, seemed to be some peripheral part of the theory of integrable systems is actually the base of these new developments (string equation, Virasoro constraints or, more general, W-constraints). We use the most general and convenient form of additional symmetries given in [4] (while the first work introducing them was [5]). Acquaintance with the general background of this theory can be made e.g. with the help of [6].

As in the first part, we also study the action of additional symmetries on the Grassmannian.

\section{Part 1. Multi-component KP and KdV (mcKdV).}

2. Definition. Let

$$
L=A \partial+u_{0}+u_{1} \partial^{-1}+\cdots
$$

where $u_{i}$ are $n \times n$ matrices, $A=\operatorname{diag}\left(a_{1}, \ldots, a_{n}\right), a_{i}$ are distinct non-zero constants. Diagonal elements of $u_{0}$ are assumed to be zero. Let $R_{\alpha}=\sum_{j=0}^{\infty} R_{j \alpha} \partial^{-j}, \alpha=1, \ldots, n$, where $R_{0 \alpha}=E_{\alpha}$, $E_{\alpha}$ is a matrix having only one non-zero element on the $(\alpha, \alpha)$ place which is equal to $1 ; R_{\alpha}$ is supposed to satisfy

$$
\left[L, R_{\alpha}\right]=0 .
$$

It can be shown that such matrices exist and their elements are differential polynomials in elements of $u_{i}$ being

$$
R_{\alpha} R_{\beta}=\delta_{\alpha \beta} R_{\alpha}, \quad \sum_{\alpha=1}^{n} R_{\alpha}=I
$$

(i.e. this is a spectral decomposition of the unity). The mcKP hierarchy (multicomponent $\mathrm{KP})$ is

$$
\partial_{k \alpha} L=\left[\left(L^{k} R_{\alpha}\right)_{+}, L\right], \quad \partial_{k \alpha}=\partial / \partial t_{k \alpha}, \quad k=0,2, \ldots ; \alpha=1, \ldots, n
$$

and $t_{k \alpha}$ are the "time variables" of the hierarchy. The equations imply $\partial_{k \alpha} R_{\beta}=\left[B_{k \alpha}, R_{\beta}\right]$. Notice that $\partial_{0 \alpha} L=\left[E_{\alpha}, L\right]$.

It can be shown that the equations commute. The variables $x$ and $t_{k \alpha}$ are not independent:

$$
\partial=\sum_{\alpha} a_{\alpha}^{-1} \partial_{1 \alpha}
$$

(Greek indices always run from 1 to $n$ ).

Remark 1. Two viewpoints on the last formula are possible: i) not to introduce explicitly $x$ and to consider $\partial$ as $\sum_{\alpha} a_{\alpha}^{-1} \partial_{1 \alpha}$ by definition, ii) to consider a separate variable $x$, however, $x$ and $t_{1 \alpha}$ are involved in formulas not independently but only in combinations $t_{1 \alpha}+a_{\alpha}^{-1} x$. Here we accept the first point, in Sects. 7 and 8 the second one. Let

$$
L=\hat{w} A \partial \hat{w}^{-1}, \text { where } \hat{w}=\hat{w}(A \partial)=\sum_{0}^{\infty} w_{i}(A \partial)^{-i}, w_{0}=I
$$


Then $R_{\alpha}=\hat{w} E_{\alpha} \hat{w}^{-1}$. Put

$$
w=\hat{w}(A \partial) \exp \xi(t, z)=\hat{w}(z) \exp \xi(t, z) ; \text { where } \xi(t, z)=\sum_{k=0}^{\infty} \sum_{\alpha=1}^{n} z^{k} E_{\alpha} t_{k \alpha} .
$$

This is the Baker function; it satisfies the equations

$$
L w=z w, \text { and } \partial_{k \alpha} w=\left(L^{k} R_{\alpha}\right)_{+} w .
$$

The latter equation is equivalent to

$$
\partial_{k \alpha} \hat{w}=-\left(L^{k} R_{\alpha}\right)_{-} \hat{w} .
$$

Remark 2. Series $\hat{w}$ are defined up to a multiplication on the right by series $\sum_{0}^{\infty} a_{i} \partial^{-i}$ with constant diagonal matrices $a_{i}$. Instead of being constant they can satisfy a weaker condition $\partial a_{i}=0$, then the last equation must be written in a more general form:

$$
\partial_{k \alpha} \hat{w}=-\left(L^{k} R_{\alpha}\right)_{-} \hat{w}+\hat{w} b
$$

where $b=\sum_{1}^{\infty} b_{i} \partial^{-i}$ is a diagonal series in $\partial^{-1}$ with coefficients not depending on $x$.

Remark 3. We have $\partial_{0 \alpha} \hat{w}=-\left(R_{\alpha}-E_{\alpha}\right) \hat{w}=-\hat{w} E_{a}+E_{\alpha} \hat{w}=\left[E_{\alpha}, \hat{w}\right]$. Symmetries related to "zero" time variables $t_{0 \alpha}$ are similarity transformations with constant matrices.

3. Additional symmetries. We introduce them in a way similar to that in the scalar case (see, e.g. [6], sect. 7.8.1.).

The operator $\partial_{k \alpha}-\left(L^{k} R_{\alpha}\right)_{+}$can be represented in a "dressing" form:

$$
\begin{gathered}
\partial_{k \alpha}-\left(L^{k} R_{\alpha}\right)_{+}=\hat{w} \partial_{k \alpha} \hat{w}^{-1}+\left(\partial_{k \alpha} \hat{w}\right) \hat{w}^{-1}-\left(L^{k} R_{\alpha}\right)_{+} \\
=\hat{w} \partial_{k \alpha} \hat{w}^{-1}-L^{k} R_{\alpha}=\hat{w}\left(\partial_{k \alpha}-(A \partial)^{k} E_{\alpha}\right) \hat{w}^{-1}
\end{gathered}
$$

(using (2.2)). Thus, the hierarchy equation (2.1) is a dressing of an obvious equality $\left[\partial_{k \alpha}-(A \partial)^{k} E_{\alpha}, A \partial\right]=0$.

There is another operator commuting with $\partial_{k \alpha}-(A \partial)^{k} E_{\alpha}$. This is

$$
\Gamma=\sum_{l=1}^{\infty} \sum_{\beta=1}^{n} t_{l \beta} l(A \partial)^{l-1} E_{\beta}
$$

Put $M=\hat{w} \Gamma \hat{w}^{-1}$. Dressing the equality

$$
\left[\partial_{k \alpha}-(A \partial)^{k} E_{\alpha}, \Gamma\right]=0
$$

we obtain

$$
\partial_{k \alpha} M=\left[\left(L^{k} R_{\alpha}\right)_{+}, M\right] .
$$

Together with (2.1) this implies more general equality

$$
\partial_{k \alpha}\left(M^{k} L^{m}\right)=\left[\left(L^{k} R_{\alpha}\right)_{+}, M^{k} L^{m}\right] .
$$


In order to define a one-parameter group of additional symmetry, take some variable $t_{l, m, \alpha}^{*}$ (the asterisk superscript will distinguish variables of additional symmetries) and consider an equation

$$
\partial_{l, m, \alpha}^{*} \hat{w}=-\left(M^{l} L^{m} R_{\alpha}\right)_{-} \hat{w}, \quad \partial_{l, m, \alpha}^{*}=\partial / \partial t_{l, m, \alpha}^{*}
$$

which is the definition of a one-parameter group of transformation of $\hat{w}$. Then

$$
\partial_{l, m, \alpha}^{*} L=-\left[\left(M^{l} L^{m} R_{\alpha}\right)_{-}, L\right], \quad \partial_{l, m, \alpha}^{*} R_{\alpha}=-\left[\left(M^{l} L^{m} R_{\alpha}\right)_{-}, R_{\alpha}\right] .
$$

This implies

$$
\begin{gathered}
\partial_{l, m, \alpha}^{*}\left(L^{k} R_{\beta}\right)_{-}=-\left[\left(M^{l} L^{m} R_{\alpha}\right)_{-},\left(L^{k} R_{\beta}\right)_{-}\right]_{-}-\left[\left(M^{l} L^{m} R_{\alpha}\right)_{-},\left(L^{k} R_{\beta}\right)_{+}\right]_{-} \\
=-\left[\left(M^{l} L^{m} R_{\alpha}\right)_{-},\left(L^{k} R_{\beta}\right)_{-}\right]-\left[M^{l} L^{m} R_{\alpha}, \partial_{k \beta}\right]_{-}=-\left[\left(M^{l} L^{m} R_{\alpha}\right)_{-},\left(L^{k} R_{\beta}\right)_{-}+\partial_{k \beta}\right] .
\end{gathered}
$$

Proposition 1. $\partial_{k \beta}$ and $\partial_{l, m, \alpha}^{*}$ commute.

$$
\begin{gathered}
{\left[\partial_{l, m, \alpha}^{*}, \partial_{k, \beta}\right] L=-\partial_{l, m, \alpha}^{*}\left[\left(L^{k} R_{\beta}\right)_{-}, L\right]+\partial_{k, \beta}\left[\left(M^{l} L^{m} R_{\alpha}\right)_{-}, L\right]} \\
=\left[\partial_{k, \beta}\left(M^{l} L^{m} R_{\alpha}\right)_{-}-\partial_{l, m, \alpha}^{*}\left(L^{k} R_{\beta}\right)_{-}, L\right]+\left[\left(L^{k} R_{\beta}\right)_{-},\left[\left(M^{l} L^{m} R_{\alpha}\right)_{-}, L\right]\right] \\
-\left[\left(M^{l} L^{m} R_{\alpha}\right)_{-},\left[\left(L^{k} R_{\beta}\right)_{-}, L\right]\right]=\left[\partial_{k, \beta}\left(M^{l} L^{m} R_{\alpha}\right)_{-}-\partial_{l, m, \alpha}^{*}\left(L^{k} R_{\beta}\right)_{-}, L\right] \\
+\left[\left[\left(L^{k} R_{\beta}\right)_{-},\left(M^{l} L^{m} R_{\alpha}\right)_{-}\right], L\right] \\
=\left[\partial_{k, \beta}\left(M^{l} L^{m} R_{\alpha}\right)_{-}-\partial_{l, m, \alpha}^{*}\left(L^{k} R_{\beta}\right)_{-}+\left[\left(L^{k} R_{\beta}\right)_{-},\left(M^{l} L^{m} R_{\alpha}\right)_{-}\right], L\right] \\
=\left[\left[\partial_{k, \beta}+\left(L^{k} R_{\beta}\right)_{-},\left(M^{l} L^{m} R_{\alpha}\right)_{-}\right]-\partial_{l, m, \alpha}^{*}\left(L^{k} R_{\beta}\right)_{-}, L\right] \\
=\left[\left[\partial_{k, \beta}+\left(L^{k} R_{\beta}\right)_{-},\left(M^{l} L^{m} R_{\alpha}\right)_{-}\right]+\left[\left(M^{l} L^{m} R_{\alpha}\right)_{-}, \partial_{k, \beta}+\left(L^{k} R_{\beta}\right)_{-}\right], L\right]=0 .
\end{gathered}
$$

If they commute on $L$ then they commute on the whole differential algebra generated by coefficients of $L$.

Proposition 2. The Lie algebra of operators $\partial_{l, m, \alpha}^{*}$ is isomorphic to the Lie algebra generated by $-z^{l} \partial_{z}^{m} E_{\alpha}$ where $\partial_{z}=\partial / \partial z$ (or $-z^{m} \partial_{z}^{l} E_{\alpha}$, which is the same).

This is what they call $W_{\infty}$-algebra.

Proof. First we find the following commutation relations:

$$
\begin{gathered}
{[L, M]=\hat{w}\left[A \partial, \sum_{l, \beta} t_{l \beta}(A \partial)^{l-1} E_{\beta}\right] \hat{w}^{-1}=\hat{w} \frac{1}{n} \sum_{\beta} a_{\beta} \cdot a_{\beta}^{-1} E_{\beta} \hat{w}^{-1}=1,} \\
{\left[R_{\alpha}, M\right]=\hat{w}\left[E_{\alpha}, \sum_{l, \beta} t_{l \beta}(A \partial)^{l-1} E_{\beta}\right] \hat{w}^{-1}=0 .}
\end{gathered}
$$

Then we prove

Lemma. The correspondence $\partial_{l, m, \alpha}^{*} \mapsto M^{l} L^{m} R_{\alpha}$ is an anti-isomorphism of Lie algebras.

(One may not identify $\partial_{0, m, \alpha}^{*}$ and $\partial_{m, \alpha}$ in this lemma: they act on $M$ in different ways, $\partial_{0, m, \alpha}^{*} M=-\left[\left(L^{m} R_{\alpha}\right)_{-}, M\right]$ while $\partial_{m, \alpha} M=\left[\left(L^{m} R_{\alpha}\right)_{+}, M\right]$; the action of these operators on $L$ coincides. The reason of this distinction is the explicit dependence $M$ of $t_{k \alpha}$ ). 
Proof of the lemma. For the simplicity of notations, let $M^{l} L^{m} R_{\alpha}=a_{l m \alpha}$. We have (a circle around a subscript "minus",$\ominus$, means that this subscript can be omitted)

$$
\begin{gathered}
{\left[\partial_{l, m, \alpha}^{*}, \partial_{k, n, \beta}^{*}\right] L=-\partial_{l, m, \alpha}^{*}\left[\left(a_{k n \beta}\right)_{-}, L\right]-((l, m, \alpha) \Leftrightarrow(k, n, \beta))} \\
=\left[\left[\left(a_{l m \alpha}\right)_{-}, a_{k n \beta}\right]_{-}, L\right]+\left[\left(a_{k n \beta}\right)_{-},\left[\left(a_{l m \alpha}\right)_{-}, L\right]\right]-((l, m, \alpha) \Leftrightarrow(k, n, \beta)) \\
=\left[\left[\left(a_{l m \alpha}\right)_{-},\left(a_{k n \beta}\right)_{-}\right]_{\ominus}, L\right]+\left[\left[\left(a_{l m \alpha}\right)_{\ominus},\left(a_{k n \beta}\right)_{+}\right]_{-}, L\right] \\
+\left[\left[\left(a_{k n \beta}\right)_{-},\left(a_{l m \alpha}\right)_{-}\right], L\right]+\left[\left(a_{l m \alpha}\right)_{-},\left[\left(a_{k n \beta}\right)_{-}, L\right]\right] \\
-\left[\left[\left(a_{k n \beta}\right)_{-}, a_{l m \alpha}\right]_{-}, L\right]-\left[\left(a_{l m \alpha}\right)_{-},\left[\left(a_{k n \beta}\right)_{-}, L\right]\right] \\
=\left[\left[a_{l m \alpha}, a_{k n \beta}\right]_{-}, L\right]=-\left[\left[a_{k n \beta}, a_{l m \alpha}\right]_{-}, L\right] .
\end{gathered}
$$

The rest follows from the fact that the commutation relation for $M$ and $L$ is the same as for $z$ and $\partial_{z}$, that $\left\{R_{\alpha}\right\}$ commute with both $L$ and $M$, and that $\left\{R_{\alpha}\right\}$ is a spectral decomposition of the unity. The proposition is proven.

Remark. The isomorphism stated in the proposition 2 is not simply an abstract isomorphism: operators $-z^{m} \partial_{z}^{l} E_{\alpha}$ are exactly how $\partial_{l, m, \alpha}^{*}$ act on the Grassmannian (see sect. 8).

4. Reductions. The hierarchy and additional symmetries admit reductions to submanifolds of operators $L$ such that $L^{h}$ is a purely differential operator, i.e. $L^{h}=L_{+}^{h}$, operators $L^{k}$ being understood as $\left(L_{+}^{h}\right)^{k / h}$. This means that the right hand sides of the equations giving the hierarchy and additional symmetries can be written in terms of $\left(L_{+}^{h}\right)^{k / h}$ modulo $L_{-}^{h}$. This implies that, if initially $L_{-}^{h}=0$, it will not arise in the process of motion along the trajectories of the hierarchy or of the additional symmetries.

We shall call this reduction the $h$ th $\mathrm{mcKdV}$ reduction. For this reduction we have

$$
\sum_{\alpha} \partial_{(l h) \alpha} L=-\left[L^{l h}, L\right]=0, \quad l=1,2, \ldots
$$

One of the most important reduction (considered in [2]) is for $h=1$ :

$$
L=A \partial+u_{0} .
$$

In the scalar case this reduction is trivial.

There is also a reduction of a different kind. We can assume that operators $L$ are formally skew-symmetric, $L^{*}=-L$ (where $\left.\left(f \partial^{k}\right)^{*}=(-\partial)^{k} f\right)$ which corresponds to unitary $\hat{w}$. Then $R_{\alpha}$ are self-adjoint. We also can consider only real $L$.

For example, let the matrix dimension $n$ be 2 . Consider both reductions, $L$ being real skew-symmetric and $h=1$. Then $L=A \partial+u_{0}$ where $A$ can be chosen as $\operatorname{diag}(1,-1)$ and

$$
L=\left(\begin{array}{ll}
\partial & -v \\
v & -\partial
\end{array}\right)
$$

Changing the frame we obtain

$$
L=\frac{1}{2}\left(\begin{array}{cc}
1 & 1 \\
1 & -1
\end{array}\right)\left(\begin{array}{ll}
\partial & -v \\
v & -\partial
\end{array}\right)\left(\begin{array}{cc}
1 & 1 \\
1 & -1
\end{array}\right)=\left(\begin{array}{cc}
0 & \partial+v \\
\partial-v & 0
\end{array}\right) .
$$


This is the operator discussed in [2].

5. String equation. Let $l \geq 1$. The equation (3.4) implies

$$
\partial_{l, m, \alpha}^{*} L^{h}=-\left[\left(M^{l} L^{m} R_{\alpha}\right)_{-}, L^{h}\right]=-\left[M^{l} L^{m} R_{\alpha}, L^{h}\right]+\left[\left(M^{l} L^{m} R_{\alpha}\right)_{+}, L^{h}\right] .
$$

We shall assume the system being reduced to purely differential operators $L^{h}$. The first term of the r.h.s. of the above formula is

$$
\sum_{i=1}^{l}\left(\begin{array}{l}
h \\
i
\end{array}\right)\left(\begin{array}{l}
l \\
i
\end{array}\right) i ! M^{l-i} L^{h-i+m} R_{\alpha} .
$$

If $l=1$ and $m=-h+1$ then the sum reduces to one term which is a constant, $h$. Summing over $\alpha$, we have

$$
\partial_{1,-h+1}^{*} L^{h}=h+\left[\left(M L^{-h+1}\right)_{+}, L^{h}\right] .
$$

where $\partial_{l, m}^{*}=\sum_{\alpha} \partial_{l, m, \alpha}^{*}$. Evidently, $\partial_{l, m}^{*} L=-\left[\left(M^{l} L^{m}\right)_{-}, L\right]$.

The string equation is $\partial_{1,-h+1}^{*} L^{h}=0$, or, in an equivalent form,

$$
\left[L^{h},\left(M L^{-h+1}\right)_{+}, L^{h}\right]=h .
$$

In the case of the reduction $h=1$ it is simply

$$
\left[L, M_{+}\right]=1
$$

(which is an equation, in contrast to the identity $[L, M]=1$ ).

6. Grassmannian. Definition of the Grassmannian. Let $H$ be $L^{2}\left(S, \mathbf{C}^{n}\right)$, a space of series $v(z)=\sum_{-\infty}^{\infty} v_{k} z^{k}$ where $v_{k} \in \mathbf{C}^{n},|z|=1$, let $H_{+}$and $H_{-}$be spaces of truncated series: $H_{+}=\left\{\sum_{0}^{\infty}\right\}, H_{-}=\left\{\sum_{-\infty}^{-1}\right\} ; H=H_{+} \oplus H_{-}$. Let $p_{ \pm}$be natural projections of $H$ onto these subspaces. We shall think of vectors as vector-rows.

The Grassmannian, Gr, is the set of all subspaces $V \subset H$ enjoying the properties: $\left.p_{+}\right|_{V}$ is a one-to-one correspondence (i.e. we restrict the definition to a generic case), and $p_{-} \mid V$ is an operator of a trace class. We say that a matrix function belongs to $V$ if all its rows do. Let $g(t, z)=\exp \xi=\exp \sum_{k=0}^{\infty} \sum_{\alpha=1}^{n} z^{k} E_{\alpha} t_{k \alpha}$. Let $\mathbf{g}^{-1}$ be a transformation of the space $H$ :

$$
\mathbf{g}^{-1}: H \rightarrow H, v \mapsto v g^{-1} \text {. }
$$

If $V \in \mathrm{Gr}$ then for almost all $t$ the subspace $V g^{-1} \in \mathrm{Gr}$.

The Baker function corresponding to an element $V \in \mathrm{Gr}$, denoted as $w_{V}(t, z)$, is determined by conditions i) for every set of variables $t=\left(t_{k \alpha}\right) w_{V}$ belongs to $V$ as a function of $z$, ii) $p_{+}\left(w_{V} g^{-1}\right)=1$. Together the conditions mean that $w_{V}(t, z)$ is the unique element of $V$ of the form

$$
w_{V}(t, z)=\left(1+\sum_{1}^{\infty} w_{i} z^{-i}\right) g(t, z) \equiv \hat{w}_{V}(t, z) g(t, z) .
$$

Proposition 1. The Baker function $w_{V}(t, z)$ is a Baker function of the mcKP hierarchy defined in a formal sense in the section 2. 
The proof can be found in [3].

Proposition 2. If $z^{h} V \subset V$ for an element of the Grassmannian then $L^{h}$ is a purely differential operator.

Proof. We have

$$
\sum_{\alpha}\left(L^{h} R_{\alpha}\right)_{+} w_{V}-z^{h} w_{V}=\sum_{\alpha} \partial_{h \alpha} w_{V}-z^{h} w_{V}=\sum_{\alpha} \partial_{h \alpha} \hat{w}_{V} \cdot g(t, z) .
$$

Now, $\partial_{h \alpha} w_{V}$ belong to $V$ as derivatives with respect to parameters, so does $z^{h} w_{V}$ by the assumption, which implies that $\sum_{\alpha} \partial_{h \alpha} \hat{w}_{V} \cdot g(t, z) \in V$. By the above mentioned uniqueness this gives $\sum_{\alpha} \partial_{h \alpha} \hat{w}_{V}=0$ and $\sum_{\alpha}\left(L^{h} R_{\alpha}\right)_{+} w_{V}-z^{h} w_{V}=0$ i.e. $\left(L^{h}\right)_{+} w_{V}-z^{h} w_{V}=0$. On the other hand, $L^{h} w_{V}-z^{h} w_{V}=0$ which yields $\left(L^{h}\right)_{-} w_{V}=0$ and $L_{-}^{h}=0$, as required.

In particular, $L$ is a differential operator, $L=A \partial+u_{0}$ if the corresponding element of the Grassmannian enjoys the property $z V \subset V$.

7. z-operators and the Grassmannian. If a reader is not interested in the problem how the additional symmetries act on the Grassmannian, he can skip this and the next sections and go directly to the section 9 dealing with action of additional symmetries on $\tau$-functions and with Virasoro constraints; the more so, as for the special case of $\partial_{1, m, \alpha}^{*}$ with $m \leq 0$, responsible for the string equation, the main result of these two sections, the proposition of Sect. 8, will be proven independently and much easier in Sect. 9. 2).

In this and the next sections we use separate variables $x$ and $t_{1 \alpha}$ (see Remark 1 in Sect.

In the same way as in the scalar case one can introduce "z-operators", following Mulase [6]:

$$
G=G\left(\partial_{z}, z\right)=\sum_{j \geq 0, i \leq i_{0}} a_{i j} z^{i} \partial_{z}^{j}, \quad \partial z=\partial / \partial z
$$

where the coefficients $a_{i j}$ are matrices now. Operators

$$
G=1+\sum_{j \geq 0, i<0} a_{i j} z^{i} \partial_{z}^{j}
$$

are called monic.

The ring of $z$-operators is isomorphic to the ring of $\Psi$ DO under the mapping

$$
z \mapsto-A \partial, \quad \partial_{z} \mapsto A^{-1} x
$$

since $\left[\partial_{z}, z\right]=\left[A^{-1} x,-A \partial\right]=1$.

In the vector case there are the same two Mulase's theorems as in the scalar case:

Proposition 1. If $V \in G r$ is an element of a Grassmannian then there is a monic zoperator $G$ such that $V^{T}=G H_{+}^{T}$. (The superscript $T$ is here for transpose; elements of $V$ and $H_{+}$are vector-rows and those of $V^{T}$ and $H_{+}^{T}$ are vector-columns). 
Proposition 2. If a $z$-operator preserves $H_{+}$then it must involve only non-negative powers of $z$.

The $z$-operators enable one to find direct relations between elements of the Grassmannian and the dressing operator $\hat{w}$.

Let $V$ be an element of the Grassmannian. Then, generically, $V \exp (-\xi(t, z))$ is also an element of the Grassmannian. The proposition 1 implies that there is a monic operator $\Psi\left(t, A \partial_{z},-A^{-1} z\right)$ such that

$$
e^{-\xi(t, z)} V^{T}=\Psi H_{+}^{T} .
$$

For every $v \in V$ there is some $h_{+} \in H_{+}$such that $\exp (-\xi) v^{T}=\Psi h_{+}^{T}$ i.e. $h_{+}^{T}=\Psi^{-1} \exp (-\xi) v^{T}$. Differentiating this with respect to $t_{k \alpha}$ we have

$$
\begin{gathered}
\partial_{k \alpha} h_{+}^{T}=-\Psi^{-1}\left(\partial_{k \alpha} \Psi\right) \Psi^{-1} \exp (-\xi) v^{T}-\Psi^{-1} z^{k} E_{\alpha} \exp (-\xi) v^{T} \\
=-\Psi^{-1}\left(\partial_{k \alpha} \Psi\right) h_{+}^{T}-\Psi^{-1} z^{k} E_{\alpha} \Psi h_{+}^{T} .
\end{gathered}
$$

Put $L^{*}=\Psi^{-1} z \Psi$ and $R_{\alpha}^{*}=\Psi^{-1} E_{\alpha} \Psi$. Then the obtained equation can be rewritten as

$$
\left[-\Psi^{-1}\left(\partial_{k \alpha} \Psi\right)-\left(\left(L^{*}\right)^{k} R_{\alpha}^{*}\right)^{T}\right] h_{+}^{T}=\partial_{k \alpha} h_{+}^{T} .
$$

The right-hand side belongs to $H_{+}$, then so does the left-hand side. The element $h_{+} \in$ $H_{+}$is an arbitrary element of $H_{+}$; applying the proposition 2 we see that $-\Psi^{-1}\left(\partial_{k \alpha} \Psi\right)-$ $\left.\left(\left(L^{*}\right)^{k} R_{\alpha}^{*}\right)\right)_{-}=0$ and $\partial_{k \alpha} \Psi=-\Psi\left(\left(L^{*}\right)^{k} R_{\alpha}^{*}\right)_{-}$. Passing to $\Psi D O$ we get the same equality and $L^{*}=\Psi^{-1}(-A \partial) \Psi, R_{\alpha}^{*}=\Psi^{-1} E_{\alpha} \Psi$.

Let us pass now to conjugate operators which means that all matrices should be replaced by transposes, $\partial$ by $-\partial$, and all factors written in the inverse order. Conjugation is denoted by a star, $*$. Let

$$
\hat{w}(t, x, \partial)=\Psi^{*}(t, x, \partial), L=\left(L^{*}\right)^{*}, R_{\alpha}=\left(R_{\alpha}^{*}\right)^{*} .
$$

Then we arrive at the equations

$$
\partial_{k \alpha} \hat{w}=-\left(L^{k} R_{\alpha}\right)_{-} \hat{w}, L=\hat{w} A \partial \hat{w}^{-1}, R_{\alpha}=\hat{w} E_{\alpha} \hat{w}^{-1} .
$$

This is nothing but the equation of the hierarchy. Thus, we constructed a dressing operator corresponding to $V \in \mathrm{Gr}$.

Notice that in our construction variables $t_{k \alpha}$ and $x$ where independent; the hierarchy equations show that $\hat{w}$ depends on the variables $x$ and $t_{1 \alpha}$ only in combinations $t_{1 \alpha}+a_{\alpha}^{-1} x$.

8. Additional symmetries and the Grassmannian. We are going to obtain the action of additional symmetries (3.3) on elements of the Grassmannian.

All operators involved in (3.3) depend on $x$ in combinations $t_{1 \alpha}+a_{\alpha}^{-1} x$ (see Sect. 2, Remark 1). In particular, $M=\hat{w} \Gamma \hat{w}^{-1}$ where

$$
\Gamma=\sum_{l=1}^{\infty} \sum_{\beta=1}^{n} t_{l \beta} l(A \partial)^{l-1} E_{\beta}+\sum_{\beta=1}^{n} a_{\beta}^{-1} x E_{\beta}=\sum_{l=1}^{\infty} \sum_{\beta=1}^{n} t_{l \beta} l(A \partial)^{l-1} E_{\beta}+A^{-1} x .
$$

Let us take the conjugated equation:

$$
\partial_{l, m, \alpha}^{*} \Psi=-\Psi\left(\left(L^{*}\right)^{m}\left(M^{*}\right)^{l} R_{\alpha}^{*}\right)_{-}
$$


where, as in the previous section, $\Psi=\hat{w}^{*}$.

Now, pass from the $\Psi$ DO to the $z$-operators. A relation $\exp (-\xi) v^{T}=\Psi h_{+}^{T}$ connects an arbitrary element $v$ of $V \in \mathrm{Gr}$ with an element $h_{+} \in H_{+}$. Act with $\partial_{l, m, \alpha}^{*}$ on both the sides of this equality:

$$
\begin{gathered}
\partial_{l, m, \alpha}^{*} \exp (-\xi) v^{T}=-\Psi\left(\left(L^{*}\right)^{m}\left(M^{*}\right)^{l} R_{\alpha}^{*}\right)_{-} h_{+}^{T}+\Psi \partial_{l, m, \alpha}^{*} h_{+}^{T} \\
=-\Psi\left(\left(L^{*}\right)^{m}\left(M^{*}\right)^{l} R_{\alpha}^{*}\right) h_{+}^{T}+\Psi g_{+}^{T}
\end{gathered}
$$

where $g_{+}^{T}=\left(\left(L^{*}\right)^{m}\left(M^{*}\right)^{l} R_{\alpha}^{*}\right)_{+} h_{+}^{T}+\partial_{l, m, \alpha}^{*} h_{+}^{T}$ is an element of $H_{+}$. Now,

$$
\begin{gathered}
\partial_{l, m, \alpha}^{*} \exp (-\xi) v^{T}=-z^{m}\left(\sum_{l=1}^{\infty} \sum_{\beta=1}^{n} t_{l \beta} l(A \partial)^{l-1} E_{\beta}+\partial_{z}\right)^{l} E_{\alpha} \Psi h_{+}^{T}+\Psi g_{+}^{T} \\
=-z^{m}\left(\sum_{l=1}^{\infty} \sum_{\beta=1}^{n} t_{l \beta} l(A \partial)^{l-1} E_{\beta}+\partial_{z}\right)^{l} E_{\alpha} \exp (-\xi) v^{T}+\exp (-\xi) v_{1}^{T}
\end{gathered}
$$

where $v_{1}$ is an element of $V$. Now, notice that $\left(\sum_{l=1}^{\infty} \sum_{\beta=1}^{n} t_{l \beta} l(A \partial)^{l-1} E_{\beta}+\partial_{z}\right) \exp (-\xi)=0$, hence

$$
\left(\sum_{l=1}^{\infty} \sum_{\beta=1}^{n} t_{l \beta} l(A \partial)^{l-1} E_{\beta}+\partial_{z}\right)^{l}\left(\exp (-\xi) v^{T}\right)=\exp (-\xi) \partial_{z}^{l} v^{T} .
$$

Using this formula and canceling $\exp (-\xi)$ in the previous equation we obtain

$$
\partial_{l, m, \alpha}^{*} v^{T}=-z^{m} \partial_{z}^{l} E_{\alpha} v^{T}+v_{1}^{T} .
$$

Since the action of an infinitesimal operator on an element of the Grassmannian is defined as a mapping $V \rightarrow H / V$ (see e.g. [1]), the second term does not play any role, and the operator $\partial_{l, m, \alpha}^{*}$ acts on elements of the Grassmannian as the operator $-z^{m} \partial_{z}^{l} E_{\alpha}$. Thus, we have proven:

Proposition. Additional symmetries $\partial_{l, m, \alpha}^{*}$ act on transposed elements of the Grassmannian (i.e. on vector-columns) as operators $-z^{m} \partial_{z}^{l} E_{\alpha}$. (Their action on vector-rows differs: multiplication on $E_{\alpha}$ is on the right).

If an operator $\mathbf{E}_{\alpha}$ acting on elements $v \in V$ as $\mathbf{E}_{\alpha} v=v E_{\alpha}$ is introduced then the above proposition can be formulated as: $\partial_{l, m, \alpha}$ act on the elements of the Crassmannian as operators $-z^{m} \partial^{l} \mathbf{E}_{\alpha}$.

9. Action of additional symmetries on the Baker and the $\tau$-functions. Like the Baker functions of the usual KP, Baker functions of mcKP can be expressed in terms of $\tau$-functions (see, e.g., [3]). The significance of this fact is that infinitely many variables, coefficients of the Baker function, are replaced by only one function, in the case of KP, and $n^{2}$ functions for mcKP.

In the latter case a $\tau$-function is a matrix $\mathrm{T}=\left\{\tau_{\alpha \beta}\right\}$ with equal diagonal elements $\tau_{\alpha \alpha}=\tau$, and the relation between the Baker and the $\tau$-functions is the following:

$$
\hat{w}_{\alpha \alpha}(t, z)=\frac{\tau\left(\ldots, t_{s \gamma}-\delta_{\gamma \beta} \cdot 1 / s z^{s}, \ldots\right)}{\tau(t)},
$$


and

$$
\hat{w}_{\alpha \beta}(t, z)=z^{-1} \frac{\tau_{\alpha \beta}\left(\ldots, t_{s \gamma}-\delta_{\gamma \beta} \cdot 1 / s z^{s}, \ldots\right)}{\tau(t)}, \alpha \neq \beta .
$$

(Attention: only those time variables $t_{s \gamma}$ are shifted whose index $\gamma$ coincides with the number of the column, $\gamma=\beta$ ).

We start with the action of additional symmetries on the Baker functions and then transfer it to the $\tau$-functions, using (9.1) and (9.2). For simplicity we are doing this for the most important case of symmetries $\partial_{1, m, \alpha}^{*}$, related to the string equation.

For the dressing operator $\hat{w}=\hat{w}(A \partial)$ we have

$$
\partial_{1, m, \alpha}^{*} \hat{w}=-\left(M L^{m} R_{\alpha}\right)_{-} \hat{w}=-\left\{\hat{w} \sum_{k=1}^{\infty} k t_{k \alpha}(A \partial)^{k+m-1} E_{\alpha} \hat{w}\right\}_{-} \hat{w} .
$$

Here we concentrate on the case $m \leq 0$. The right hand side of the last equation is a sum of three terms, with negative, zero and positive $k+m-1$, the first of them is

$$
\mathrm{N}=-\hat{w} \sum_{1}^{-m} k t_{k \alpha}(A \partial)^{k+m-1} E_{\alpha}=-\left[\hat{w}, t_{1 \alpha}\right](A \partial)^{m} E_{\alpha}-\sum_{1}^{-m} k t_{k \alpha} \hat{w}(A \partial)^{k+m-1} E_{\alpha} .
$$

(This term is absent for $m=0$ ). The second is

$$
\begin{gathered}
\mathrm{Z}=-\left\{\hat{w}(-m+1) t_{-m+1, \alpha} E_{\alpha} \hat{w}^{-1}\right\}_{-} \hat{w} \\
=-\hat{w}(-m+1) t_{-m+1, \alpha} E_{\alpha}+(-m+1) t_{-m+1, \alpha} E_{\alpha} \hat{w} \\
=-(-m+1)\left[\hat{w}, t_{-m+1, \alpha} E_{\alpha}\right]=-(-m+1)\left[\hat{w}, t_{-m+1, \alpha}\right] E_{\alpha} \delta_{m, 0}-(-m+1) t_{-m+1, \alpha}\left[\hat{w}, E_{\alpha}\right] .
\end{gathered}
$$

The third:

$$
\begin{gathered}
P=\sum_{-m+2}^{\infty} k t_{k \alpha} \partial_{k+m-1, \alpha} \hat{w} \\
=-\left[\hat{w}, t_{1 \alpha}\right] E_{\alpha} \delta_{m, 0}+(-m+1) t_{-m+1, \alpha} \partial_{0 \alpha} \hat{w} .
\end{gathered}
$$

Now, notice:

$$
\begin{gathered}
\text { i) } \hat{w}(A \partial) \exp \left(A^{-1} x z\right)=\hat{w}(z) \exp \left(A^{-1} x z\right), \quad \text { ii) }\left[A \partial, t_{1 \alpha}\right] E_{\alpha}=E_{\alpha}, \\
\text { iii) } \hat{w}(A \partial) t_{1 \alpha} \exp \left(A^{-1} x z\right) E_{\alpha}=\left\{t_{1 \alpha} \hat{w}(z)+\partial_{z} \hat{w}(z)\right\} \exp \left(A^{-1} x z\right) E_{\alpha},
\end{gathered}
$$

or

$$
\left[\hat{w}(A \partial), t_{1 \alpha}\right] \exp \left(A^{-1} x z\right) E_{\alpha}=\partial_{z} \hat{w} \exp \left(A^{-1} x z\right) E_{\alpha} .
$$

Applying the operator $\partial_{1, m, \alpha}^{*} \hat{w}$ to $\exp \left(A^{-1} x z\right)$ and canceling $\exp \left(A^{-1} x z\right)$ we get

Proposition 1. The action of additional symmetries $\partial_{1, m, \alpha}^{*}$ with $m \leq 0$ on $\hat{w}$ is given by

$$
\begin{aligned}
\partial_{1, m, \alpha}^{*} \hat{w}(z)= & -z^{m} \partial_{z} \hat{w}(z) E_{\alpha}-\sum_{1}^{-m} k t_{k \alpha} z^{k+m-1} \hat{w}(z) E_{\alpha} \\
& +\sum_{-m+1}^{\infty} k t_{k \alpha} \partial_{k+m-1, \alpha} \hat{w}(z)
\end{aligned}
$$


or, in coordinates,

$$
\begin{aligned}
\partial_{1, m, \alpha}^{*} \hat{w}_{\gamma \beta}(z)= & -z^{m} \partial_{z} \hat{w}_{\gamma \beta}(z) \delta_{\alpha \beta}-\sum_{1}^{-m} k t_{k \alpha} z^{k+m-1} \hat{w}_{\gamma \beta}(z) \delta_{\alpha \beta} \\
& +\sum_{-m+1}^{\infty} k t_{k \alpha} \partial_{k+m-1, \alpha} \hat{w}_{\gamma \beta}(z)
\end{aligned}
$$

Remark. Like the action of the KP operators on $\hat{w}$ (see remark 2 at the end of Sect. 2 ), the action of additional symmetries is defined up to terms $\hat{w} b$ where $b=\sum_{1}^{\infty} b_{i} \partial^{-i}$ is a series with constant diagonal matrices $b_{i}$.

One can also obtain an action of $\partial_{1, m, \alpha}^{*}$ on the Baker function $w=\hat{w} \exp \xi(t, z)$. It is easy to see that

$$
\partial_{1, m, \alpha}^{*} w=-z^{m} \partial_{z} w E_{\alpha}+\sum_{-m+1}^{\infty} k t_{k \alpha} \partial_{k+m-1, \alpha} w .
$$

If $w$ is $w_{V}$ i.e. the Baker function determined by an element $V$ of the Grassmannian, then $w \in V$, the last terms of the above equality also belongs to $V$, and $\partial_{1, m, \alpha}^{*}$ acts on $w$ as the operator $-z^{m} \partial_{z} \mathbf{E}_{\alpha}$ modulo $V$, where $\mathbf{E}_{\alpha} w$, by definition, is $w E_{\alpha}$.

Values of $w$ for all $t$ span the subspace $V$. Action of $\partial_{1, m, \alpha}^{*}$ on an element of the Grassmannian $V$ is the action of this operator on elements of $V$ modulo $V$, i.e. the mapping $V \rightarrow H / V$ (see e.g. [1]). Therefore, we have independently proved the proposition of Sect. 8: Additional symmetries $\partial_{1, m, \alpha}^{*}$ act on elements of the Grassmannian as operators $-z^{m} \partial_{z} \mathbf{E}_{\alpha}$.

In [1] it was shown how, using the relations of the type (9.1) and (9.2), to transfer the action of $\partial^{*}$ s from $\hat{w}$ to $\tau$. It was done in both ways: the equivalence between some actions of $\partial^{*}$ on $\hat{w}$ and on $\tau$ was proven. Now, for brevity, we do this in one way: show that some given action of $\partial^{*}$ on $\tau_{\alpha \beta}$ implies (9.3).

Proposition 2. The action of additional symmetries $\partial_{1, m, \alpha}^{*}$ with $m \leq 0$ on $\left(\tau_{\gamma \beta}\right)$ is given by

$$
\partial_{1, m, \alpha}^{*} \tau_{\gamma \beta}=\left(\sum_{k=-m+1}^{\infty} k t_{k \alpha} \partial_{k+m-1, \alpha}+\frac{1}{2} \sum_{k+l=-m+1} k l t_{k \alpha} t_{l \alpha}\right) \tau_{\gamma \beta}+c \tau_{\gamma \beta}
$$

where $t_{k \alpha}$ are arguments of this element $\tau_{\gamma \beta}$ (Eqs (9.1)and (9.2) show that some of them can be shifted, and then we should replace $t$ in this formula by a shifted argument).

Proof. Let $\tilde{\tau}_{\alpha \beta}=\tau_{\alpha \beta}\left(\ldots, t_{s \gamma}-\delta_{\gamma \beta} \cdot 1 / s z^{s}, \ldots\right)$. Eq (9.4) implies that

$$
\partial_{1, m, \alpha}^{*} \tilde{\tau}_{\gamma \alpha}=\left[\sum_{k=-m+1}^{\infty} k\left(t_{k \alpha}-\frac{1}{k z^{k}}\right) \partial_{k+m-1, \alpha}+\frac{1}{2} \sum_{k+l=-m+1} k l\left(t_{k \alpha}-\frac{1}{k z^{k}}\right)\left(t_{l \alpha}-\frac{1}{l z^{l}}\right)\right] \tilde{\tau}_{\gamma \alpha}+c \tilde{\tau}_{\gamma \alpha}
$$

We have

$$
\partial_{1, m, \alpha}^{*} \hat{w}_{\gamma \beta}=\tau^{-1} \partial_{1, m, \alpha}^{*} \tilde{\tau}_{\gamma \beta}-\tilde{\tau}_{\gamma \beta} \tau^{-2} \partial_{1, m, \alpha}^{*} \tau .
$$

First we consider $\beta \neq \alpha$.

$$
\partial_{1, m, \alpha}^{*} \tilde{\tau}_{\gamma \beta}=\left(\sum_{k=-m+1}^{\infty} k t_{k \alpha} \partial_{k+m-1, \alpha}+\frac{1}{2} \sum_{k+l=-m+1} k l t_{k \alpha} t_{l \alpha}\right) \tilde{\tau}_{\gamma \beta}+c \tilde{\tau}_{\gamma \beta}
$$


and, as it is easy to see, $\partial_{1, m, \alpha}^{*} \hat{w}_{\gamma \beta}=\sum_{k=-m+1}^{\infty} k t_{k \alpha} \partial_{k+m-1, \alpha} \hat{w}_{\gamma \beta}$ that coincides with the required by $(9.3)$.

Now, let $\beta=\alpha$. Then

$$
\begin{aligned}
\partial_{1, m, \alpha}^{*} \hat{w}_{\gamma \alpha}= & \tau^{-1}\left[\sum_{k=-m+1}^{\infty} k\left(t_{k \alpha}-\frac{1}{k z^{k}}\right) \partial_{k+m-1, \alpha}+\frac{1}{2} \sum_{k+l=-m+1} k l\left(t_{k \alpha}-\frac{1}{k z^{k}}\right)\left(t_{l \alpha}-\frac{1}{l z^{l}}\right)\right] \tilde{\tau}_{\gamma \alpha} \\
& -\tilde{\tau}_{\gamma \alpha} \tau^{-2}\left(\sum_{k=-m+1}^{\infty} k t_{k \alpha} \partial_{k+m-1, \alpha}+\frac{1}{2} \sum_{k+l=-m+1} k l t_{k \alpha} t_{l \alpha}\right) \tau \\
= & \sum_{k=-m+1}^{\infty} k t_{k \alpha} \partial_{k+m-1, \alpha} \tilde{\tau}_{\gamma \alpha}-\sum_{k+l=-m+1} t_{k \alpha} z^{-l} \hat{w}_{\gamma \alpha}-\frac{m}{2} z^{m-1} \hat{w}_{\gamma \alpha} .
\end{aligned}
$$

If one takes into account that

$$
\tau^{-1} \sum_{k=-m+1}^{\infty} z^{-k} \partial_{k+m-1, \alpha} \tilde{\tau}_{\gamma \alpha}=z^{m} \partial_{z} \tilde{\tau}_{\gamma \alpha} / \tau=z^{m} \partial_{z} \hat{w}_{\gamma \alpha}
$$

and that the term $(m / 2) z^{m-1} \hat{w}_{\gamma \alpha}$ is irrelevant according to the above remark, this coincides with (9.3). This proves our proposition.

Thus, the string equation is equivalent to the requirement that

$$
\sum_{\alpha=1}^{n}\left(\sum_{k=-m+1}^{\infty} k t_{k \alpha} \partial_{k+m-1, \alpha}+\frac{1}{2} \sum_{k+l=-m+1} k l t_{k \alpha} t_{l \alpha}\right) \tau_{\gamma \beta}+c_{\alpha \beta} \tau_{\gamma \beta}=0 \quad \gamma, \beta=1, \ldots, n .
$$

This is the so-called Virasoro constraint. It is clear that also higher Virasoro constraints can be written in the same manner as in the scalar case (see, e.g. [1]). We skip this.

\section{Part 2. Modified KdV (mKdV).}

10. Definition. Let $v_{1}, \ldots, v_{n}$ be independent generators of a differential algebra $\mathcal{A}_{v}$. We shall also use $v_{k}$ with any integer subscript $k$ assuming that $v_{k+n}=v_{k}$. Put

$$
L_{i}:=\left(\partial+v_{i}\right) \ldots\left(\partial+v_{n}\right)\left(\partial+v_{1}\right) \ldots\left(\partial+v_{i-1}\right)=\left(\partial+v_{i}\right) \ldots\left(\partial+v_{i+n-1}\right)
$$

and $B_{k}^{[i]}=\left(L_{i}^{k / n}\right)_{+}, k \in \mathbf{Z}$, which is a $k$ th order operator. Each $L_{i}$ can also be represented as

$$
L_{i}=\partial^{n}+u_{1}^{[i]} \partial^{n-1}+\ldots+u_{n}^{[i]}
$$

The coefficients $\left\{u_{k}^{[i]}\right\}, k=1, \ldots, n$ are elements of $\mathcal{A}_{v}$

$$
u_{k}^{[i]}=F_{k}\left(v_{i}, \ldots, v_{i+n-1}\right), k=1, \ldots, n .
$$

These functions define the Miura transformation. They determine an embedding of the differential algebra $\mathcal{A}_{u^{[i]}}$ of all differential polynomials in $u_{k}^{[i]}$ into $\mathcal{A}_{v}$,

$$
\mathcal{A}_{u[i]} \subset \mathcal{A}_{v}
$$


(If we wish $u_{1}^{[i]}=0$ then it must be $v_{1}+\ldots+v_{n}=0$ ).

For each $L_{i}$ we can construct its KdV hierarchy

$$
\partial_{k} L_{i}=\left[B_{k}^{[i]}, L_{i}\right], k=1,2, \ldots ; \partial_{k}=\partial / \partial t_{k} .
$$

Lemma. $B_{k}^{[i]}\left(\partial+v_{i}\right)-\left(\partial+v_{i}\right) B_{k}^{[i+1]}$ (where, by definition, $\left.B_{k}^{[n+1]}=B_{k}^{[1]}\right)$ are zero-order differential operators.

Proof. Starting from an obvious relation $L_{i}\left(\partial+v_{i}\right)=\left(\partial+v_{i}\right) L_{i+1}$ we derive $L_{i}^{k / n}\left(\partial+v_{i}\right)=$ $\left(\partial+v_{i}\right) L_{i+1}^{k / n}$ whence

$$
B_{k}^{[i]}\left(\partial+v_{i}\right)-\left(\partial+v_{i}\right) B_{k}^{[i+1]}=-\left(L_{i}^{k / n}\right)_{-}\left(\partial+v_{i}\right)+\left(\partial+v_{i}\right)\left(L_{i+1}^{k / n}\right)_{-} .
$$

The r.h.s. is a zero-order $\Psi D O$ hence so is the l.h.s. On the other hand, this is a differential operator.

Corollary. The system of equation

$$
\partial_{k} v_{i}=B_{k}^{[i]}\left(\partial+v_{i}\right)-\left(\partial+v_{i}\right) B_{k}^{[i+1]}, i=1, \ldots, n
$$

makes sense.

This system (for every fixed $k$ ) is called a modified KdV equation (mKdV).

Proposition. The equation (10.3) is an extention to $\mathcal{A}_{v}$ of every equation (10.2) given on $\mathcal{A}_{u^{[i]}}$ (i.e. for each fixed $\left.i=1, \ldots, n\right)$.

Remark. Differential algebras $\mathcal{A}_{u^{[i]}}$ do not coincide i.e. elements of one of them are not, generally, differential polynomials of another. A transition of one of them to another via $\mathcal{A}_{v}$ (i.e. by integrating the Miura formulas with respect to $v_{k}$ ) is the Bäcklund transformation discovered by Adler [8].

Proof of the proposition. Let $\partial_{k}$ be given on the generators $v_{k}$ of $\mathcal{A}_{v}$ by (10.3). Then

$$
\begin{gathered}
\partial_{k} L_{i}=\partial_{k}\left(\partial+v_{i}\right) \ldots\left(\partial+v_{i+n-1}\right)=\sum_{l=1}^{i+n-1}\left(\partial+v_{i}\right) \ldots\left(\partial+v_{l-1}\right)\left(B_{k}^{[l]}\left(\partial+v_{l}\right)\right. \\
\left.-\left(\partial+v_{l}\right) B_{k}^{[l+1]}\right)\left(\partial+v_{l+1}\right) \ldots\left(\partial+v_{i+n-1}\right)=B_{k}^{[i]} L_{i}-L_{i} B_{k}^{[i]}=\left[B_{k}^{[i]}, L_{i}\right] .
\end{gathered}
$$

All the equations (10.2), for any $i$, are the same $\mathrm{KdV}$ system but they differ by their embedding into $\mathcal{A}_{v}$. If a solution $u_{k}$ to the $\mathrm{KdV}$ equation is given we can take it for $u_{k}^{[i]}$ and solve Eq.(10.1) with respect to $v_{k}$. Solutions depend on some arbitrary constants which may depend on the time variable. The previous proposition means that the constants can be chosen properly to make $v_{k}$ satisfy the mKdV equation. (It suffices to find $v_{k}$ in an initial moment $t=t_{0}$ and then take them as initial conditions for the mKdV system. If we return from $v_{k}(t)$ to $u_{k}(t)$ solving Eq.(10.1) in the opposite direction, this solution to $\mathrm{KdV}$ must coincide with the original since they coincide at the initial moment). 
Thus $n$ solutions of the KdV equation correspond to any solution of mKdV. They are, of course, not independent, but closely connected by the equation

$$
L_{i}\left(\partial+v_{i}\right)=\left(\partial+v_{i}\right) L_{i+1} \quad\left(L_{n+1}=L_{1}\right) .
$$

We call them compatible.

11. $\tau$-functions. Let us have $\mathrm{n}$ compatible solutions to $\mathrm{KdV}$ equation, $L_{i}$. Operators $L_{i}^{1 / n}$ are solutions to KP hierarchy, and a $\tau$-function corresponds to each of them, $\tau_{i}$.

Proposition. The corresponding solution to $m K d V$ can be expressed in terms of $\tau_{i}$ as

$$
v_{i}=\partial \log \left(\tau_{i+1} / \tau_{i}\right) \quad\left(\tau_{n+1}=\tau_{1}\right)
$$

Proof. We represent all the $L_{i}$ in the form of dressing;

$$
L_{i}=\hat{w}^{[i]}(\partial) \partial^{n}\left(\hat{w}^{[i]}(\partial)\right)^{-1}, \quad \hat{w}^{[i]}(\partial)=\sum_{0}^{\infty} w_{k}^{[i]} \partial^{-k}, w_{0}^{[i]}=1
$$

$\hat{w}^{[i]}$ are unique to within multiplication on the right by constant series $\sum_{0}^{\infty} c_{k}^{[i]} \partial^{-k}, c_{0}=1$. Equations

$$
\hat{w}^{[i]} \partial^{n}\left(\hat{w}^{[i]}\right)^{-1}\left(\partial+v_{i}\right)=\left(\partial+v_{i}\right) \hat{w}^{[i+1]} \partial^{n}\left(\hat{w}^{[i+1]}\right)^{-1}
$$

imply $\left[\partial^{n},\left(\hat{w}^{[i]}\right)^{-1}\left(\partial+v_{i}\right) \hat{w}^{[i+1]}\right]=0$. Then $\left(\hat{w}^{[i]}\right)^{-1}\left(\partial+v_{i}\right) \hat{w}^{[i+1]}=\partial \cdot \sum_{0}^{\infty} c_{k}^{[i]} \partial^{-k}, c_{k}^{[i]}=$ const, $c_{0}^{[i]}=1$.

Using the freedom of multiplication of $\hat{w}^{[i]}$ on the right by constant series we can obtain a simpler formula $\left(\hat{w}^{[i]}\right)^{-1}\left(\partial+v_{i}\right) \hat{w}^{[i+1]}=\partial$ and

$$
\partial+v_{i}=\hat{w}^{[i]} \partial\left(\hat{w}^{[i+1]}\right)^{-1}=\left(1+w_{1}^{[i]} \partial^{-1}+\ldots\right) \partial\left(1-w_{1}^{[i+1]} \partial^{-1}+\ldots\right)
$$

which implies $v_{i}=w_{1}^{[i]}-w_{1}^{[i+1]}$. On the other hand, the Baker function can be expressed in terms of the $\tau$-function

$$
\hat{w}^{[i]}(z)=\tau^{[i]}\left(\ldots, t_{k}-1 / k z^{k}, \ldots\right) / \tau^{[i]}(t)
$$

whence $w_{1}^{[i]}=-\partial_{1} \tau^{[i]} / \tau^{[i]}=-\partial \log \tau^{[i]}$. Now the required equality is obvious.

Now we are going to give a construction of a set of compatible $\tau$-functions within the framework of Segal-Wilson's theory.

The original idea was given by Wilson [9].

We know that solutions to the KdV hierarchy are related to elements of a submanifold of the Grassmannian, $G r^{(n)} \subset G r$ defined by the property $z^{n} V \subset V$ for $V \in G r^{(n)}$.

Now, we have $n$ operators $L_{i}$ corresponding to one solution of $\mathrm{mKdV}$. Therefore, our main objects now will be $n$-tuples of elements of the Grassmannian, $V=\left(V_{1}, \ldots, V_{n}\right)$. Of course, they cannot be independent since $L_{i}$ are not. We will require the following property:

$$
z V_{i} \subset V_{i+1}, \quad i=1, \ldots, n ; \quad\left(V_{n+1}=V_{1}\right) .
$$


Example. Let $H$ be the space $L_{2}$ on the circle $|z|=1$, and

$$
V_{i}=\left\{f(z)=\sum_{-N}^{\infty} f_{k} z^{k} \mid f\left(a_{l}\right)=\epsilon^{-i} \alpha_{l} f\left(\epsilon a_{l}\right) ; l=1, \ldots, N, \epsilon^{n}=1\right\} .
$$

Functions $f$ are supposed to be prolonged into the circle and $a_{l}$ are distinct points, $0<\left|a_{l}\right|<$ 1 , while $\alpha_{l}$ are arbitrary non-zero numbers. It is easy to see that all properties are satisfied.

Eq.(6) implies that $z^{n} V_{i} \subset V_{i}$, thus, $V_{i} \in \mathrm{Gr}^{(n)}$, and there are corresponding $\tau$-functions $\tau_{i}$ and Baker functions $w_{V_{i}}=w_{V, i}$.

Proposition. There are operators $\partial+v_{k}$ such that

$$
\left(\partial+v_{k}\right) w_{V, k+1}=z w_{V, k} .
$$

Proof. Let $g=\exp \xi(t, z)$ where $\xi(t, z)=\sum_{1}^{\infty} t_{k} z^{k}$. By the definition of a Baker function

$$
w_{V, k+1}=\left(1+a_{1} z^{-1}+\ldots\right) g \in V_{k+1},
$$

then

$$
\begin{aligned}
\partial w_{V, k+1} & =\left(z+a_{1}+\ldots\right) g \in V_{k+1} \\
w_{V, k} & =\left(1+b_{1} z^{-1}+\ldots\right) g \in V_{k}, \\
z w_{V, k} & =\left(z+b_{1}+\ldots\right) g \in z V_{k} \subset V_{k+1}
\end{aligned}
$$

whence

$$
\partial w_{V, k+1}-z w_{V, k}-\left(a_{1}+b_{1}\right) w_{V, k+1}=O\left(z^{-1}\right) g \in V_{k+1},
$$

therefore this quantity is zero (since the Baker function is unique), and $v_{k}=-\left(a_{1}+b_{1}\right)$.

Corollary. If $w_{V, k}=\hat{w}_{V, k}$ g, where $\hat{w}_{V, k}=\sum_{0}^{\infty} w_{k, i} \partial^{-i}$, then

$$
\left(\partial+v_{k}\right) \cdot \hat{w}_{k+1, W}=\hat{w}_{k, W} \cdot \partial .
$$

This is nothing but the compatibility condition for mKdV. It implies

$$
\left(\partial+v_{i}\right) \ldots\left(\partial+v_{i+n-1}\right) \hat{w}_{i}=\hat{w}_{i} \partial^{n}
$$

i.e. $L_{i}=\hat{w}_{i} \partial^{n} \hat{w}_{i}^{-1}$. The formula (11.1) with $\tau_{i}=\tau_{i, W}, \tau_{i+1}=\tau_{i+1, W}$ provides us with a solution to $\mathrm{mKdV}$.

12. $\mathbf{m K d V}$ as a reduction of $\mathbf{m c K d V}$. The relation (10.2), (10.3), (10.4) and others can be represented in a matrix form (see also [11]). Let

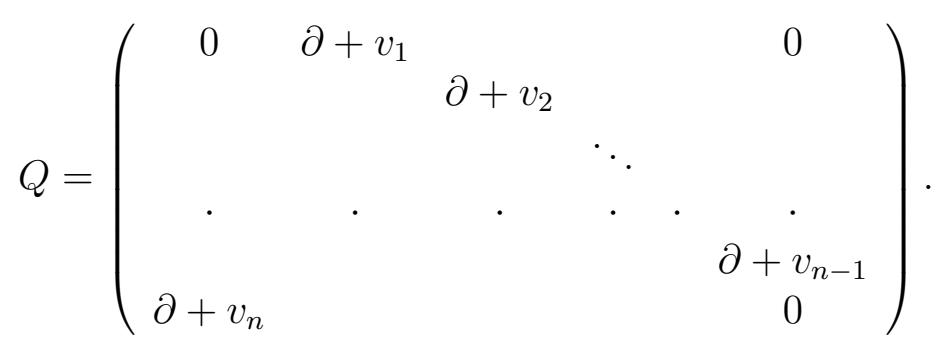


Then

$$
Q^{n}=L=\operatorname{diag}\left(L_{1}, \ldots, L_{n}\right)
$$

where $L_{i}$ were defined above. (This is, actually, the Miura transformation). Let $B_{k}=$ $\operatorname{diag}\left(B_{k}^{[1]}, \ldots, B_{k}^{[n]}\right)$. Then Eq. (10.2) and (10.3) can be written as

$$
\partial_{k} L=\left[B_{k}, L\right], \quad \partial_{k} Q=\left[B_{k}, Q\right] .
$$

(these are $\mathrm{KdV}$ and $\mathrm{mKdV}$, correspondingly). The operator $Q$ looks like the operator (4.1) of the mcKdV hierarchy $h=1$. There are two distinctions: firstly, we have matrices $u_{0}$ of a very special form and, secondly, $A$ is no more diagonal but $A_{i j}=\delta_{i+1, j}$ (recall periodicity: $i+n$ is identified with $i$ ). There are various possibilities to deal with such $A$. For example, changing the basis, we can reduce $A$ to a diagonal form $\operatorname{diag}\left(1, \epsilon, \ldots, \epsilon^{n-1}\right)$ where $\epsilon$ is an $n$th root of the unit. We can also preserve the matrix $A$ as it is, replacing spectral projectors $E_{\alpha}$ by

$$
E_{\alpha}=(1 / n) \sum_{r} \epsilon^{-\alpha r} A^{r}
$$

However, this project also has its disadvantage: the matrices $E_{\alpha}$ are complex. The best of all, and we do this further, is to preserve the same $A$, but, instead of the spectral projectors, just to use powers $A^{-\alpha}$ of $A$.

We have to restrict some mcKdV flow to the matrices $Q=(\partial+V) A$ where $V=\operatorname{diag}\left(v_{1}, \ldots, v_{n}\right)$. The submanifold of these matrices among all matrices $\Lambda=A \partial+U$ will be denoted by $\mathbf{Q}$.

Let us consider dressing: $A \partial+U=\hat{w} \partial A \hat{w}^{-1}$ i.e. $(A \partial+U) \hat{w}=\hat{w} \partial A, \hat{w}=1+O\left(\partial^{-1}\right)$. The dressing operator $\hat{w}$ is not unique: it can be multiplied on the right by monic series in $\partial^{-1}$ of the type $\sum a_{j k} \partial^{-j} A^{k}$, coefficients are scalar constants.

Define basic mcKdV flows as

$$
\partial_{m \alpha} \Lambda=-\left[\left(\Lambda^{m} R_{\alpha}\right)_{-}, \Lambda\right]
$$

where $R_{\alpha}=\hat{w} A^{-\alpha} \hat{w}^{-1}$. It is easy to see that $\partial_{11}=\partial$.

Lemma. If $\Lambda=Q \in \mathbf{Q}$ then a diagonal matrix can be chosen as $\hat{w}$.

Proof. Rewrite the dressing equations in coordinates:

$$
\left(\partial+v_{i}\right) \hat{w}_{i+1, j}=\hat{w}_{i, j-1} \partial .
$$

The equality $L^{n} \hat{w}=\hat{w} \partial^{n}$ yields $\left(\partial+v_{i}\right) \ldots\left(\partial+v_{i+n-1}\right) \hat{w}_{i j}=\hat{w}_{i j} \partial^{n}$. This implies that $\hat{w}_{i j} \partial^{n} \hat{w}_{i j}^{-1}$ does not depend on $j, \hat{w}_{i j} \partial^{n} \hat{w}_{i j}^{-1}=\hat{w}_{i j_{1}} \partial^{n} \hat{w}_{i j_{1}}^{-1}$ whence $\hat{w}_{i j_{1}}^{-1} \hat{w}_{i j}$ commutes with $\partial^{n}$ and is a constant (series), $c_{i j j_{1}}$. We have now $\hat{w}_{i j}=c_{i j j_{1}} \hat{w}_{i j_{1}}$. Evidently, $c_{i, j, j+1} c_{i, j+1, j+2}=c_{i, j, j+2}$ etc. Another relation between these coefficients we obtain replacing in (12.3) $\hat{w}_{i+1, j}$ by $c_{i+1, j, j+1} \hat{w}_{i+1, j+1}$ and $\hat{w}_{i, j-1}$ by $c_{i, j-1, j} \hat{w}_{i j}$. This gives $c_{i+1, j, j+1}=c_{i, j-1, j}$. Two obtained relations together mean that coefficients can be represented as $c_{i, j, j+r}=c_{i-j, r}$. Then $\hat{w}_{i j}=c_{i-j, r} \hat{w}_{i, j+r}$ and, in particular, $\hat{w}_{i j}=c_{i-j, i-j} \hat{w}_{i i}$ which means that $\hat{w}=\hat{\hat{w}} \sum c_{r, r} A^{r}$ where $\hat{\hat{w}}$ is the diagonal part of $\hat{w}$. This proves the lemma, since there is a freedom to multiply $\hat{w}$ by $\left(\sum c_{r, r} A^{r}\right)^{-1}$. 
Proposition. The $m K d V$ flow $\partial_{k}$ on $\mathbf{Q}$ can be extended to the mcKdV flow $\partial_{k k}$ on the manifold of all operators $\Lambda=\partial A+U$, and variables $t_{k}$ can be identified with $t_{k k}$.

Proof. Given an mKdV flow $\partial_{k} Q=\left[L_{+}^{k / n}, Q\right]$ where $L=Q^{n}$. Let us "undress" operators $\Lambda=\partial A+U: \Lambda=\partial A+U=\hat{w} \partial A \hat{w}^{-1}$. The operator $\hat{w}$ can be chosen so that being restricted to operators $Q$ it becomes diagonal (above lemma). Take the following mcKdV flow: $\partial_{k k} \Lambda=\left[\left(\Lambda^{k} R_{k}\right)_{+}, \Lambda\right]$. Restriction of this flow to operators $Q$ is $\partial_{k k} Q=\left[\left(Q^{k} R_{k}\right)_{+}, Q\right]$. We have $Q^{k} R=\hat{w} A^{k} \partial^{k} A^{-k} \hat{w}^{-1}=\hat{w} \partial^{k} \hat{w}^{-1}$. We also have $L=Q^{n}=\hat{w} \partial^{n} \hat{w}^{-1}$. Then $L^{k / n}=\hat{w} \partial^{k} \hat{w}^{-1}$ (we use here the fact that $\hat{w}$ is a diagonal matrix since, by definition, $L^{k / n}$ is a diagonal operator whose $n$th power is $\left.L^{n}\right)$. Thus, $Q^{k} R_{k}$ can be replaced by $L^{k / n}$ and the mcKdV equation becomes $\partial_{k k} Q=\left[\left(L^{k / n}\right)_{+}, Q\right]$ i.e. it coincides with the given flow, if the variable $t_{k k}$ of the mcKdV hierarchy is identified with the variable $t_{k}$ of the $\mathrm{mKdV}$ hierarchy. This proves the proposition.

Thus, the flows $\partial_{k k}$ respect $\mathbf{Q}$ where they coincide with $\partial_{k}, \mathrm{mKdV}$ flows. The others, $\partial_{k \alpha}$, with $k \neq \alpha$, are transverse to $\mathbf{Q}$.

Remark. An element of the vector Grassmannian corresponding to a solution of mcKdV that reduces to $Q$ is a direct sum of elements $V_{i}$ of the scalar Grassmannian we were talking about in the last section.

13. $\mathrm{mKdV}$, additional symmetries, and the string equation. The situation is the following. Additional symmetries act on all operators $L_{i}$ but it is not clear whether they act in a compatible way (see above) and, thus, can be transferred on $\mathrm{mKdV}$ (and we believe they cannot). However, if there is an embedding of $\mathrm{mKdV}$ operators $Q$ into $\mathrm{mcKdV}$ operators $\Lambda=\partial A+U$ one can consider the additional symmetry flows on their whole manifold. They commute with mcKdV flows, this is exactly why they are symmetries, but they do not preserve the submanifold of operators $Q$. Nevertheless, if an operator $Q$ is independent of some additional time variable, then it satisfies the mcKdV string equation, and this equation remains invariant under the $\mathrm{mKdV}$ flow (which is, in fact a restricted mcKdV flow).

Let us see what is this string equation like. It was written in Sect. 5; now we have $h=1$, $Q$ plays the role of $L, M$ is $\hat{w} \Gamma \hat{w}^{-1}$ with $\Gamma=\sum_{l=1}^{\infty} \sum_{\beta=1}^{n} l t_{l \beta}(A \partial)^{l-1} A^{-\beta}$, and $A$ is the matrix $\delta_{i, i+1}$.

Consider the additional symmetry

$$
\partial_{1,-n+1,0}^{*} Q=-\left[\left(M Q^{-n+1}\right)_{-}, Q\right] .
$$

We have

$$
[Q, M]=\hat{w}\left[A \partial, \sum_{l=1}^{\infty} \sum_{\alpha} l t_{l \alpha}(A \partial)^{l-1} A^{-\alpha}\right] \hat{w}^{-1}=\hat{w}\left[A \partial_{11}, \sum_{l=1}^{\infty} \sum_{\alpha} l t_{l \alpha}(A \partial)^{l-1} A^{-\alpha}\right] \hat{w}^{-1}=1 .
$$

Therefore this symmetry can be rewritten as

$$
\partial_{1,-n+1,0}^{*} Q=Q^{-n+1}+\left[\left(M Q^{-n+1}\right)_{+}, Q\right]=\left(1+\left[\left(M Q^{-n+1}\right)_{+} Q^{n-1}, Q\right]\right) Q^{-n+1} .
$$

The right hand side of this equation does not belong to $\mathbf{Q}$ (The operator contains negative powers of $\partial$ ), thus, this additional symmetry cannot be reduced to $\mathbf{Q}$. 
Proposition 1. The additional symmetry (13.1) induces additional symmetries $\partial_{1,-n+1}^{*}$ on the $K d V$ operators $L_{i}$ (see [1]).

Proof. The last equation implies

$$
\partial_{1,-n+1,0}^{*} Q^{n}=n+\left[\left(M Q^{-n+1}\right)_{+}, Q^{n}\right] .
$$

Now,

$$
M Q^{-n+1}=\hat{w} \sum_{l, \alpha} l t_{l \alpha}(A \partial)^{l-1} A^{-\alpha}(A \partial)^{-n+1} \hat{w}^{-1}
$$

Let us freeze all the variables: $t_{l \alpha}=0$ except for those that preserve $\mathbf{Q}$ i.e. with $l=\alpha$. Then on $\mathbf{Q}$ we have

$$
M Q^{-n+1}=\hat{w} \sum_{l} l t_{l l} \partial^{l-1} \partial^{-n+1} \hat{w}^{-1}
$$

which coincides with $M L^{-n+1 / n}$ where $M=\hat{w} \sum_{l} l t_{l} \partial^{l-1} \hat{w}^{-1}, \hat{w}$ being a diagonal operator. The resulting equation coincides with $\partial_{1,-n+1}^{*} L=\left[\left(M L^{-n+1 / n}\right)_{+}, L\right]+n$ which is the direct sum of symmetries $\partial_{1,-n+1}^{*}$ acting on the operators $L_{1}, \ldots, L_{n}$. Exactly this was stated.

Definition. The $m K d V$ string equation is $\partial_{1,-n+1,0}^{*} Q=0$ or

$$
\left[\left(M Q^{-n+1}\right)_{+} Q^{n-1}, Q\right]=-1 .
$$

As a direct corollary of the above proposition we have

Proposition 2. The $m K d V$ string equation (13.2) induces the $K d V$ string equation

$$
\left[\left(M L^{-n+1 / n}\right)_{+}, L\right]=-n
$$

or, better to say, a string equation for each of operators $L_{i}$.

Another useful formula:

$$
\partial_{1,-n+1,0}^{*} Q=Q^{-n+1}+\sum_{l \geq n} \sum_{\alpha} l t_{l \alpha} \partial_{l-n, \alpha} Q
$$

or, preserving only the variables $t_{l l}$,

$$
\partial_{1,-n+1,0}^{*} Q=Q^{-n+1}+\sum_{l>n} l t_{l l} \partial_{l-n, l-n} Q .
$$

For the KdV operators this is

$$
\partial_{1,-n+1}^{*} L=n+\sum_{l>n} l t_{l} \partial_{l-n} L
$$

\section{References.}

1. Dickey, L. A., Additional symmetries of KP, Grassmannian, and the string equation, Preprint, 1992, hep-th 9204092. 
2. Anagnostopoulos, K. N., Bowick, M. J. and Schwarz, A., The solution space of the unitary matrix model string equation and the Sato Grassmannian, preprint, 1991.

3. Dickey, L. A., On Segal-Wilson's definition of the $\tau$-function and hierarchies AKNS$\mathrm{D}$ and mcKP, to be published in Proceedings of the Colloquium on Integrable Systems, Marseille, 1991.

4. Orlov, A. Yu., Shulman, E. I., Additional symmetries for integrable equations and conformal algebra representations, Lett. Math. Phys., 12, 1986, 171.

5. Chen, H. H., Lee, Y. C. and Lin, J. E., On a new hierarchy of symmetries for the Kadomtsev-Petviashvili equation, Physica D, 9D, n03, 1983, 439.

6. Dickey, L. A., Soliton equations and Hamiltonian systems, Advanced Series in Mathematical Physics, vol. 12, World Scientific, 1991.

7. Mulase, M., Category of vector bundles on algebraic curves and infinite dimensional Grassmannians, Internat. Journal of Math., 1, no3, 1990, 293.

8. Adler, M., On the Bäcklund transformation for the Gelfand-Dikii equations, Comm. Math. Physics, 80, no4,1981, 517.

9. Wilson, G., Infinite-dimensional Lie groups and algebraic geometry in soliton theory, Phil. Trans. Roy. Soc., London, A315, 1985, 393-404.

10. Hollowood, T., Miramontes, L., Pasquinucci, A. and Nappi, C., Hermitian versus anti-Hermitian 1-matrix models and their hierarchies, Nucl. Phys., B373, 247, 1992.

11. Kupershmidt, B. A. and Wilson, G., Modifying Lax equations and the second Hamiltonian structure, Invent. Math., 62, 403, 1981. 